\title{
An analysis of the thermodynamic cycles with high-temperature nuclear reactor for power generation and hydrogen co-production
}

\author{
Michat Dudek ${ }^{1, *}$ and Marek Jaszczur ${ }^{1}$ \\ ${ }^{1}$ AGH University of Science and Technology, Faculty of Energy and Fuels, Department of Fundamental \\ Research in Energy Engineering, Krakow, Poland
}

\begin{abstract}
In the present paper, numerical analysis of the thermodynamic cycle with the high-temperature nuclear gas reactor (HTGR) for electricity and hydrogen production have been done. The analysed system consists of two independent loops. The first loop is for HTGR and consists of a nuclear reactor, heat exchangers, and blower. The second loop (Rankine cycle) consist of up-to four steam turbines, that operate in heat recovery system. The analysis of the system shows that it is possible to achieve significantly higher efficiency than could be offered by traditional nuclear reactor technology (PWR and BWR). It is shown that the thermal efficiency about $52.5 \%$ it is possible to achieve when reactor works at standard conditions and steam is superheated up to $530^{\circ} \mathrm{C}$. For the cases when the steam has supercritical conditions the value of thermal efficiency is still very high and equal about $50 \%$.
\end{abstract}

\section{Introduction}

The growing demand for electricity consumption is one of the most important key indicators showing the development stages of countries. The most important challenges for world energy industry based nowadays on three main problems: how to reduce the impact of the industry on the environment, how to ensure the low cost of the energy production and how to improve the system overall performance. For companies operating in the Polish energy market, the number of challenges is much greater than companies operating in different countries where Nuclear Energy is used. Still open and very important for the Polish energy sector remain the issue of the construction of the first, Nuclear Power Plant. After the accident at the Fukushima Daiichi Nuclear Power Station, many countries have changed point of view to nuclear energy. More safety technologies with lower capital cost and higher thermal efficiency have been searched to ensure more attractive solutions in the future development of nuclear energy in industrial application. The choice of the first Polish nuclear power technology used to produce electricity should be determined to take into account nuclear safety, computer technology, and cyber security as well as materials science, and technical analysis, which allow today design and implement new solutions for nuclear energy. The new solution with high-efficiency energy conversion systems should provide a lower unit cost of

* Corresponding author: jaszczur@agh.edu.pl 
energy conversion and give new possibilities in energy and fuel production [1]. A new program for future nuclear energy systems, Generation IV has been created in an effort to provide next - generation technologies that will compete in all markets with the most cost effective technologies expected to be available over the next three decades [4]. Gas-cooled reactor systems have several fundamental features that distinguish them from the other types of reactors and provide significant advantages. In particular, the fuel is in the form of small ceramic - coated particles capable of operating at very high temperatures, the moderator is solid graphite and the coolant is helium or carbon dioxide inert for neutrons. Gas-Cooled Nuclear Reactor provides the potential for increased energy conversion efficiency and can be treated as a high-temperature heat source to different process heat applications, such as coal gasification or thermochemical hydrogen production. In contrary to the fossil fuels, hydrogen is cleaner and less harmful to the environment. By using this fuel we avoid most kinds of pollution, greenhouse gases, and acid rains. Several methods of hydrogen production are known and some of them required high-temperature heat.

A new generation of high-temperature nuclear reactors (HTR) is the most innovative concept among current advanced nuclear reactor technologies [2-4] and give us the opportunity to achieve a higher value of thermal efficiency and the possibility to the increase industrial application compared to another type of nuclear reactor and also give us the opportunity to realize large number heat consuming industrial, chemical and thermochemical process.
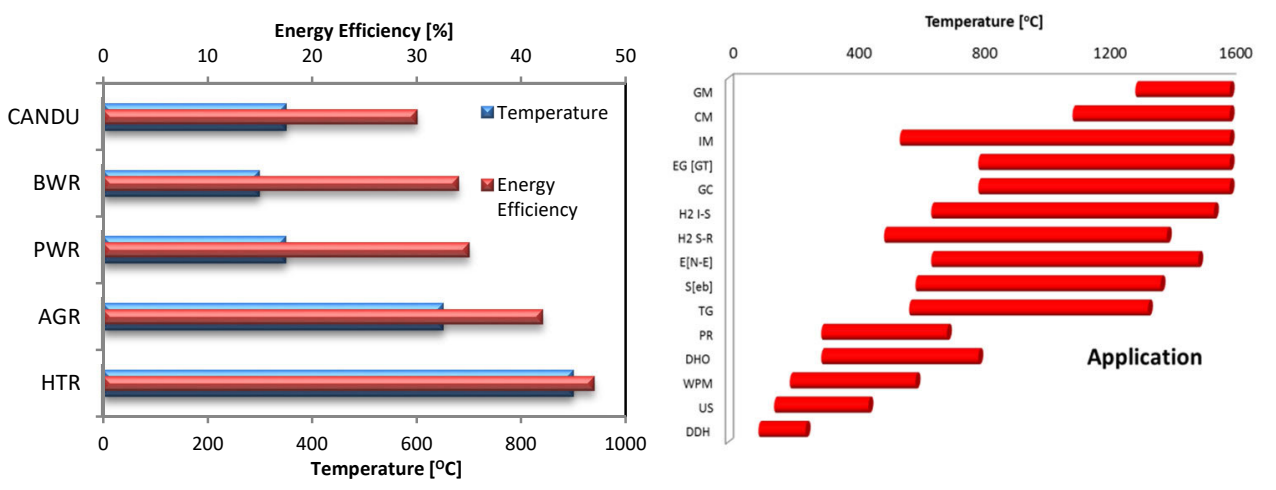

Fig. 1. (a) Operational temperatures and energy efficiency for various nuclear technologies, (b) industrial application operational temperatures

\section{High-Temperature Nuclear Reactor}

High-Temperature Nuclear Reactor is a very promising concept for using high outlet temperature for industrial purposes, at present, there is only one demonstration site in the world where such reactors are currently in construction. In Shidao Bay NPP in China the two $250 \mathrm{MW}_{\mathrm{t}}$ HTR-PM (High-Temperature Reactors-Pebble-bed Module) reactors, are able to generate power of $210 \mathrm{MW}_{\mathrm{e}}$. The construction has begun in 2012 and the operation is predicted for 2017 . The outlet temperature will be about $750^{\circ} \mathrm{C}$ and is in the low-temperature range required for efficient hydrogen production [5]. These type of reactors but designed to supply temperatures, up to $1000^{\circ} \mathrm{C}$ are called VHTRs but they are still at the research stage with no industrial use. One project launched by the United States Department of Energy (DOE) is a Next Generation Nuclear Power Plant (NGNP) which assumes designing a VHTR similar to Areva's SC-HTGR (Steam-cycle high-temperature gas-cooled reactor) with the aim of using up to $30 \%$ of a process heat to produce hydrogen. The prototype of NGNP reactor is likely to be deployed by approximately $2030[6,7]$. Another program dedicated to 
VHTRs' development was submitted by Korean Atomic Energy Research Institute (KAERI). In cooperation with China and Japan, they plan to start operation of their first VHTR, coupled with hydrogen production in 2020 [8]. With the outlet temperature of $900-1000^{\circ} \mathrm{C}$ supplied by a Very High-Temperature Reactor, the hydrogen is to be produced at efficient processes of the sulfur-iodine cycle or the electrolysis. However, with such high temperatures the problem of safe and reliable heat exchangers occurs [9]. The aim of the present work is to analyses the possibility of hydrogen production with the lower temperatures and using the nuclear systems that will be introduced to the industry in the near future. General parameters for the different type of Nuclear Reactor are described in Table 1.

Table 1. General parameters for the different type of Nuclear Reactor [10].

\begin{tabular}{|l|c|c|c|c|c|c|}
\hline & PWR & BWR & AGR & CANDU & RMBK & HTR \\
\hline Power [MW] & 1200 & 600 & 600 & 600 & 1200 & 1300 \\
\hline Fuel $\left[\%{ }^{235} \mathrm{U}\right]$ & 3,2 & 2,6 & 2,3 & 0,7 & 2 & 10 \\
\hline Dimensions of Core & $3,0 \times 3,7$ & $3,7 \times 3,7$ & $9,1 \times 8,5$ & $7,1 \times 5,9$ & $11,8 \times 7$ & $9.8 \times 6$ \\
\hline Pressure [bar] & 150 & 70 & 40 & 86 & 47 & 48 \\
\hline Outlet Temperature $\left[{ }^{\circ} \mathrm{C}\right]$ & 324 & 288 & 650 & 305 & 284 & 720 \\
\hline Steam Pressure [bar] & $4-6$ & 7 & 16 & 4,7 & 6,5 & 17,5 \\
\hline $\begin{array}{l}\text { Superheated Steam } \\
\text { Temperature }\left[{ }^{\circ} \mathrm{C}\right]\end{array}$ & $270-$ & 280 & 565 & 260 & 280 & 530 \\
\hline Thermal Efficiency $[\%]$ & 290 & & & & & \\
\hline
\end{tabular}

Actually, numerous studies have been conducted on the implementation of highTemperature Nuclear Reactors in the United States, Russia, Japan, France [6] At present on HTGR's advanced research programs are in progress in many countries. Table 2 includes basic parameters for High-Temperature Nuclear Reactor which can be used in various type of cycle [7].

Table 2. Main thermodynamic parameters for High-Temperature Nuclear Reactor [11].

\begin{tabular}{|c|c|c|c|c|}
\hline \multirow[b]{2}{*}{ Parameter } & \multicolumn{4}{|c|}{ HTR Cycle General Concepts } \\
\hline & GTMHR & $\begin{array}{c}\text { GTHTR3 } \\
00\end{array}$ & $\begin{array}{c}\text { ANTARE } \\
\text { S }\end{array}$ & $\begin{array}{c}\text { VHTR } \\
\text { NGTCC }\end{array}$ \\
\hline Power [MWth] & 600 & 600 & 600 & 350 \\
\hline Thermodynamic cycle & $\begin{array}{c}\text { Inter-Cooled } \\
\text { Recuperated } \\
\text { Brayton Cycle }\end{array}$ & $\begin{array}{c}\text { Recuperated } \\
\text { Brayton } \\
\text { Cycle }\end{array}$ & $\begin{array}{l}\text { Combined } \\
\text { Cycle }\end{array}$ & $\begin{array}{l}\text { Combined } \\
\text { Cycle }\end{array}$ \\
\hline Working fluid & $\mathrm{He}$ & $\mathrm{He}$ & $\mathrm{He} / \mathrm{N}_{2}$ & $\mathrm{He}$ \\
\hline $\begin{array}{l}\text { Reactor inlet/outlet } \\
\text { temperature }\left[{ }^{\circ} \mathrm{C}\right]\end{array}$ & $491 / 850$ & $587 / 850$ & $355 / 850$ & $400 / 950$ \\
\hline Turbine inlet temperature $\left[{ }^{\circ} \mathrm{C}\right]$ & 850 & 850 & 800 & 950 \\
\hline Reactor gas pressure [Bar] & 71 & 70 & 55 & 71 \\
\hline Compression ratio & 2.86 & 2 & 2 & 1.94 \\
\hline Plant Net Power [MWe] & 286 & 274 & $\begin{array}{c}280(80 \mathrm{GT}, \\
200 \mathrm{ST})\end{array}$ & $\begin{array}{c}180(50 \mathrm{GT}, \\
130 \mathrm{ST})\end{array}$ \\
\hline Thermal efficiency [\%] & 47.6 & 45.6 & 47.0 & 51.5 \\
\hline Number of compressor stages & 9 & 6 & 4 & 6 \\
\hline Turbine blade cooling & Uncooled & Uncooled & Uncooled & $\begin{array}{c}\text { First, two } \\
\text { stages cooled }\end{array}$ \\
\hline
\end{tabular}




\section{High-Temperature Steam-Cycle Model Description}

In the present paper, numerical analysis of high-temperature gas nuclear reactor (HTGR) thermodynamic cycle for electricity and hydrogen production have been done. The analysed system consists of two independent loops. First loop is a helium loop and is equipped with nuclear reactor, heat exchanger, and blower. The second loop is steam loop and is equipped with up to four steam turbines, that operate in heat recovery mode. The analysis of proposed system shows that is possible to achieve much higher efficiency than could be offered by traditional nuclear reactor technology (PWR 32-35\% and BWR 32-24\%).

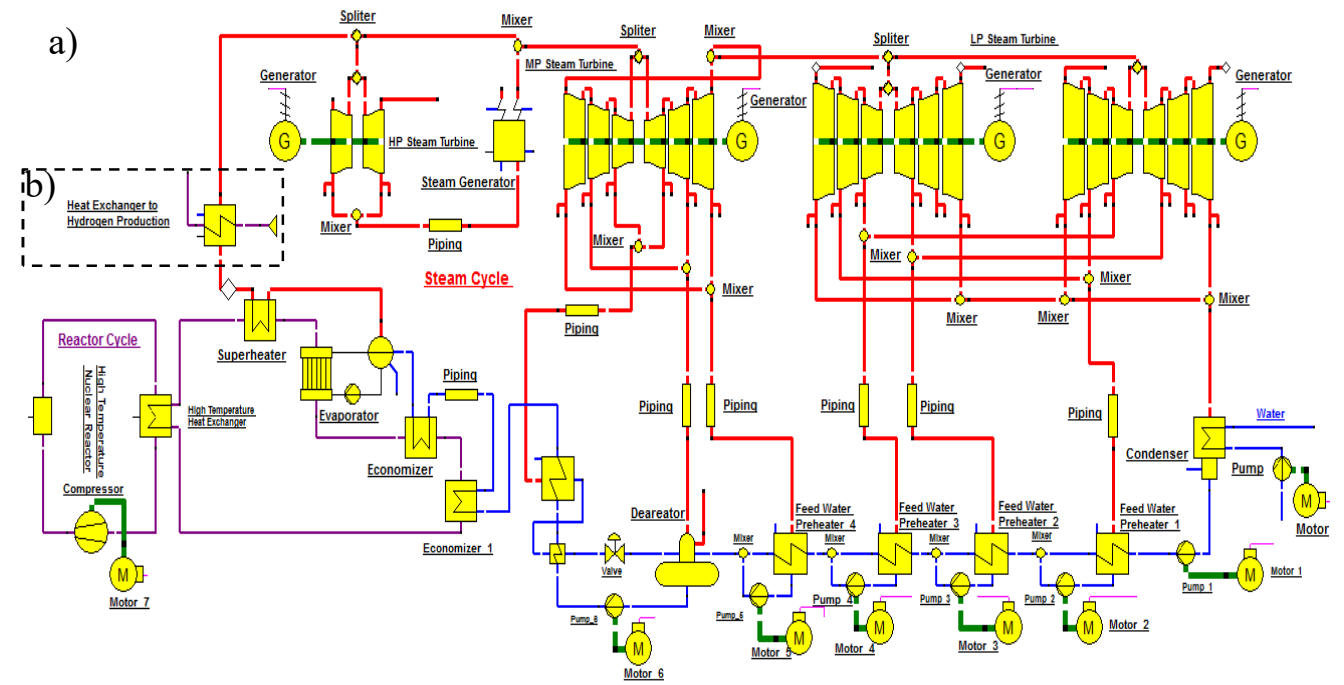

Fig. 2. The High-Temperature Nuclear Reactor thermodynamic cycle, coupled with heat recovery steam cycle: without hydrogen production (a) and with hydrogen production (b).

\section{Mathematical Model of High-Temperature Nuclear Reactor with heat recovery steam cycle}

Following equations are used to describe a mathematical model of the High-Temperature Nuclear Reactor coupled with the heat recovery steam cycle.

Reactor thermal power is calculated as follow:

$N_{R}=q_{j} \sum_{f} \phi V=q_{j} \sigma_{f} \phi m \frac{N_{A}}{A}$

$q_{j}$ - heat generated in one act cleavage, $\sigma_{f}$ - microscopic cross section $\left[\mathrm{cm}^{2}\right], \phi$ - the average neutron flux $\left[1 / \mathrm{cm}^{2} \mathrm{~s}\right], m-$ mass of fissile material $[\mathrm{kg}], N_{A}-$ Avogadro Number, $A$ mass number.

Heat recived from Nuclear Reactor:

$\dot{Q}_{R}=\dot{m}_{H e} c_{p H e}\left(T_{\text {out }}-T_{\text {in }}\right)$

$\dot{m}_{H e}$ - helium mass flow rate $[\mathrm{kg} / \mathrm{s}], c_{p H e}-$ helium specific heat $[\mathrm{kJ} /(\mathrm{kgK})]$, $T_{\text {out }}$ - reactor outlet temperature [K], $T_{\text {in }}$ - reactor inlet temperature [K]. 


\section{Heat received from high-temperature heat exchanger nuclear reactor:}

$\dot{Q}_{R 1}=\dot{m}_{H e 1} c_{p H e}\left(T_{o u t 1}-T_{i n 1}\right)$

$T_{\text {out } 1}$ - heat exchanger outlet temperature $[\mathrm{K}], T_{i n 1}$ - heat exchanger inlet temperature $[\mathrm{K}]$.

\section{Compressor work:}

$W_{\text {comp }}=\dot{m}_{H e}\left(h_{2}-h_{1}\right) / \eta_{\text {comp }} \eta_{\text {isent }}$

$h_{2}$-compressor outlet enthalpy $[\mathrm{kJ} / \mathrm{kg}], h_{1}$-inlet enthalpy to compressor $[\mathrm{kJ} / \mathrm{kg}], \eta_{\text {comp }}$ compressor mechanical efficiency, $\eta_{\text {isent }}$ - isentropic efficiency

\section{Superheater energy balance:}

$\dot{m}_{H e 1} c_{p H e}\left[T_{o u t 1}-T_{o t}\right]+\dot{m}_{p p} c_{p s p T o t}^{\operatorname{Tinp}}\left[T_{\text {inp }}-T_{o t}\right]=$

$=\dot{m}_{H e 1} c_{p H e}\left[T_{\text {out } 2}-T_{o t}\right]+\dot{m}_{p p} c_{p p p T o t}^{\text {Toutp }}\left[T_{\text {outp }}-T_{\text {ot }}\right]$

$\dot{m}_{H e 1}$ - helium mass flow rate at inlet to superheater $[\mathrm{kg} / \mathrm{s}], \quad \dot{m}_{p p}$ - mass flow rate of saturated steam/superheated steam $[\mathrm{kg} / \mathrm{s}], c_{p s p T o t}^{\text {Tinp }}$ - saturated steam specific heat $[\mathrm{kJ} /(\mathrm{kgK})]$, $c_{p H e}$-helium specific heat $[\mathrm{kJ} /(\mathrm{kgK})], c_{\text {p ppTot }}^{\text {Toutp }}$-superheated steam specific heat $\left.[\mathrm{kJ} /(\mathrm{kgK})]\right]$ $T_{\text {inp }}$ - saturated $^{-}$steam inlet temperature $[\mathrm{K}]$, $T_{\text {out } 2}$ - superheater helium outlet temperature [K], $T_{\text {outp }}$ - superheated steam outlet temperature $[\mathrm{K}], T_{o t}$-ambient temperature $[\mathrm{K}]$.

\section{Evaporator energy balance:}

$\dot{m}_{\text {Hel }} c_{p H e}\left[T_{\text {out } 2}-T_{o t}\right]+\dot{m}_{w} c_{p w 1 T o t}^{\text {Tinw }}\left[T_{i n w}-T_{o t}\right]=$

$=\dot{m}_{H e 1} c_{p H e}\left[T_{o u t 3}-T_{o t}\right]+\dot{m}_{p p} c_{p s p T o t}^{\operatorname{Tinp}}\left[T_{i n p}-T_{o t}\right]$

$\dot{m}_{w}$ - mass flow of water at inlet to evaporator $[\mathrm{kg} / \mathrm{s}], c_{p w 1}$ - water specific heat $[\mathrm{kJ} /(\mathrm{kgK})]$, $T_{i n w}$ - evaporator water inlet temperature $[\mathrm{K}], T_{\text {out } 3}$ - evaporator helium outlet temperature $[\mathrm{K}], T_{\text {inp }}$ - saturated steam outlet temperature $[\mathrm{K}], c_{\text {pspTot }}^{\text {Tinp }}$ - saturated steam specific heat $[\mathrm{kJ} /(\mathrm{kgK})]$.

\section{Economizer energy balance:}

$$
\begin{aligned}
& \dot{m}_{H e 1} c_{p H e}\left[T_{o u t 3}-T_{o t}\right]+\dot{m}_{w} c_{p w T o t}^{\text {Tinw }}\left[T_{i n w}-T_{o t}\right]= \\
& =\dot{m}_{H e 1} c_{p H e}\left[T_{o u t 4}-T_{o t}\right]+\dot{m}_{w} c_{p w 1 T o t}^{\text {Tinw }}\left[T_{i n w}-T_{o t}\right]
\end{aligned}
$$

$T_{\text {out } 4}$ - economizer helium outlet temperature $[\mathrm{K}], c_{p w}$ - water specific heat at inlet to economizer $[\mathrm{kJ} /(\mathrm{kgK})] . c_{p w 1}$ - water specific heat at outlet from economizer $[\mathrm{kJ} /(\mathrm{kgK})]$. 


\section{Economiser one energy balance:}

$$
\begin{aligned}
& \dot{m}_{H e 1} c_{p H e}\left[T_{o u t 4}-T_{o t}\right]+\dot{m}_{w} c_{p w 2 T o t}^{\text {Tinw1 }}\left[T_{i n w 1}-T_{o t}\right]= \\
& =\dot{m}_{H e 1} c_{p H e}\left[T_{i n 1}-T_{o t}\right]+\dot{m}_{w} c_{p w 1 T o t}^{\text {Tinw }}\left[T_{i n w}-T_{o t}\right]
\end{aligned}
$$

$T_{i n w 1}$-economiser water inlet temperature $[\mathrm{K}], T_{i n 1}$ - heat exchanger helium inlet temperature $[\mathrm{K}] . c_{p w 2}$ - water specific heat at inlet to economizer one $[\mathrm{kJ} /(\mathrm{kgK})], c_{p w 1}$ water specific heat at outlet from economizer one $[\mathrm{kJ} /(\mathrm{kgK})]$.

\section{HP steam turbine power:}

$$
P_{H P}=\eta_{\text {gen }} \eta_{\text {isent }} \eta_{\text {mech }} 0.5 \dot{m}_{p p}\left[h_{p p i}-h_{\text {pout }}\right]
$$

$\eta_{g e n}$ - electrical efficiency, $\eta_{\text {isent }}$ - isentropic efficiency, $\eta_{\text {mech }}$ - mechanical efficiency, $\dot{m}_{p p}$ - mass flow of superheated steam $[\mathrm{kg} / \mathrm{s}], h_{p p i}$ - steam turbine specific enthalpy at the inlet to the high-pressure $[\mathrm{kJ} / \mathrm{kg}], h_{\text {pout }}$-steam turbine specific enthalpy at the outlet $[\mathrm{kJ} / \mathrm{kg}]$.

\section{Mixer after steam generator energy balance:}

$0.5 \dot{m}_{p p} h_{p p i}+0.5 \dot{m}_{p p} h_{a s g}=\dot{m}_{p p} h_{p p i m}$

$h_{p p i}$-specific enthalpy for superheated steam after the splitter $[\mathrm{kJ} / \mathrm{kg}], h_{a s g}$ - specific enthalpy for superheated steam after steam generator $[\mathrm{kJ} / \mathrm{kg}], h_{\text {ppim }}$-enthalpy of superheated steam before MP Steam Turbine $[\mathrm{kJ} / \mathrm{kg}]$

\section{MP steam turbine power:}

$$
\begin{aligned}
& P_{M P}=\eta_{g e n} \eta_{i s e n t M P} \eta_{\text {mechMP }}\left[\dot{m}_{p p}\left[h_{p p i m}-h_{p p o i m 1}\right]\right. \\
& \left.+\left[\dot{m}_{p p}-\dot{m}_{p u 1-2}\right]\left[h_{p p o m 1}-h_{p p o i m 2}\right]+\left[\dot{m}_{p p}-\dot{m}_{\dot{m}_{p u 1-2}}-\dot{m}_{p d}\right]\left[h_{p p o m 2}-h_{p p o i m 3}\right]\right]
\end{aligned}
$$

$\eta_{g e n}$-electrical efficiency, $\eta_{\text {isent MP }}$-isentropic efficiency of steam turbine, $\eta_{\text {mechMP }}$ mechanical efficiency of steam turbine $\dot{m}_{p p}$ - mass flow at inlet to steam turbine $[\mathrm{kg} / \mathrm{s}], \dot{m}_{p p 5}$ - mass flow at first sluice inlet $[\mathrm{kg} / \mathrm{s}], \dot{m}_{p 1}$ - mass flow of steam at second sluice inlet, $h_{p p i m}$ - specific enthalpy of steam at inlet to MP steam turbine $[\mathrm{kJ} / \mathrm{kg}], h_{\text {ppoim } 1}$-specific enthalpy of steam at inlet to first sluice $[\mathrm{kJ} / \mathrm{kg}], h_{\text {ppoim } 2}$-specific enthalpy of steam at inlet to second sluice $[\mathrm{kJ} / \mathrm{kg}], h_{\text {ppoim } 3}$ - specific enthalpy of steam at outlet from MP steam turbine $[\mathrm{kJ} / \mathrm{kg}]$, $\dot{m}_{p p}$ - mass flow of superheated steam at inlet to MP steam turbine $[\mathrm{kg} / \mathrm{s}] \quad \dot{m}_{p u 1-2}$ - mass flow of saturated steam at inlet to preheater $5[\mathrm{~kg} / \mathrm{s}], \dot{m}_{p d}$ - mass flow of saturated steam at inlet to the deareator $[\mathrm{kg} / \mathrm{s}], \dot{m}_{p 4}$ - saturated steam mass flow at inlet to feed water preheater $4[\mathrm{~kg} / \mathrm{s}]$. 
MP steam turbine mass and energy balance:

$$
\begin{aligned}
& \dot{m}_{p p} h_{p p i m}=\dot{m}_{p u 1-2} h_{p p i m 1}+\dot{m}_{p d} h_{p p i m 2}+\dot{m}_{p f 4} h_{p p i m} 3+\dot{m}_{p p o} h_{p p i m 4} \\
& \dot{m}_{p p}=\dot{m}_{p u 1-2}+\dot{m}_{p d}+\dot{m}_{p f 4}+\dot{m}_{p p o}
\end{aligned}
$$

Mixers after MP Steam Turbine

$$
\begin{aligned}
& \text { Mixer_1 } \\
& \dot{m}_{p u 1}+\dot{m}_{p u 2}=\dot{m}_{p u 1-2}
\end{aligned}
$$

Mixer_2

$$
\dot{m}_{p u 3}+\dot{m}_{p u 4}=\dot{m}_{p d}
$$

Mixer_3

$$
\dot{m}_{p u 5}+\dot{m}_{p u 6}=\dot{m}_{p f 4}
$$

Mixers after first and second LP Steam Turbines

Mixer_4

$$
\dot{m}_{p u 7}+\dot{m}_{p u 13}=\dot{m}_{p f 3}
$$

Mixer_5

$$
\dot{m}_{p u 10}+\dot{m}_{p u 16}=\dot{m}_{p f 2}
$$

\section{Mixer before condenser}

$\dot{m}_{p u 9}+\dot{m}_{p u 12}+\dot{m}_{p u 15}+\dot{m}_{p u 18}=\dot{m}_{\text {wskpo }}$

$\dot{m}_{p u 1}$ - mass flow of steam from first sluice $[\mathrm{kg} / \mathrm{s}], \dot{m}_{p u 2}$ - mass flow of steam from second sluice $[\mathrm{kg} / \mathrm{s}], \dot{m}_{p u 3}$ - mass flow of steam from third sluice $[\mathrm{kg} / \mathrm{s}], \dot{m}_{p u 4}$ - mass flow of steam from fourth sluice $[\mathrm{kg} / \mathrm{s}], \dot{m}_{p u 5}$ - mass flow of steam from fifth sluice $[\mathrm{kg} / \mathrm{s}], \dot{m}_{p u 6}-$ mass flow of steam from sixth sluice $[\mathrm{kg} / \mathrm{s}], \dot{m}_{p u 7}$-mass flow of steam from seventh sluice[kg/s], $\dot{m}_{p u 13}$-mass flow of steam from the thirteenth sluice $[\mathrm{kg} / \mathrm{s}], \dot{m}_{p u 10}$-mass flow of steam from on the tenth sluice $[\mathrm{kg} / \mathrm{s}], \dot{m}_{p u 16}$ - mass flow of steam from the sixteenth sluice $[\mathrm{kg} / \mathrm{s}], \dot{m}_{p u} 9$ -mass flow of steam from ninth sluice $[\mathrm{kg} / \mathrm{s}], \dot{m}_{p u 12}$ - mass flow of steam from the twelfth sluice $[\mathrm{kg} / \mathrm{s}] \dot{m}_{p u 15}$-mass flow of steam from fifteenth sluice $[\mathrm{kg} / \mathrm{s}], \dot{m}_{p u 18}$-mass flow of steam from fourth sluice $[\mathrm{kg} / \mathrm{s}]$.

Water pump before Feed water preheater_1

$W_{p 1}=\dot{m}_{w s k p o}\left[h_{w p o}-h_{w p i n}\right]$

$h_{w p i n}$ - specific enthalpy of water at the inlet to first water pump $[\mathrm{kJ} / \mathrm{kg}]$.

Feed water preheater_1 energy balance:

$\dot{m}_{w s k p o} h_{w p o}+m_{p f 1} h_{p f 1}=\dot{m}_{p f 1} h_{p w f 1}+\dot{m}_{w s k r o} h_{w a f 1}$

$\dot{m}_{p f 1}$ - mass flow of steam at inlet to feed water preheater $1[\mathrm{~kg} / \mathrm{s}], h_{p f 1}$ - specific enthalpy of steam at inlet to feed water preheater $1[\mathrm{~kJ} / \mathrm{kg}], \dot{m}_{\text {wskpo }}$ - mass flow of water at inlet/outlet to/from feed water preheater $1[\mathrm{~kg} / \mathrm{s}], h_{w p o}$ - specific enthalpy of water at inlet to feed water preheater $1[\mathrm{~kJ} / \mathrm{kg}], h_{p w f 1}$ - specific enthalpy of water at outlet from feed water preheater 1 $[\mathrm{kJ} / \mathrm{kg}], h_{\text {waf } 1}$ - specific enthalpy of water after feed water preheater $1[\mathrm{~kJ} / \mathrm{kg}]$.

\section{Water pump before Feed water preheater_2}

$W_{p 2}=\dot{m}_{p f 1}\left[h_{w p f 2}-h_{w p f 1}\right]$

Mixer before Feed water preheater_2 energy balance. 


$$
\dot{m}_{p f 1} h_{p w f 2}+\dot{m}_{w s k p o} h_{w a f 1}=\dot{m}_{w f 2} h_{w f 2}
$$

Feed Water Preheater_2 energy balance:

$\dot{m}_{p f 2} h_{p f 2}+\dot{m}_{w f 2} h_{w f 2^{\prime}}=\dot{m}_{p f 2} h_{w f 2}+\dot{m}_{w f 2} h_{w a f 2}$

$\dot{m}_{p f 2}$ - mass flow of steam at inlet to feed water preheater $2[\mathrm{~kg} / \mathrm{s}], h_{p f 2}$ - specific enthalpy of steam at inlet to feed water preheater $2[\mathrm{~kJ} / \mathrm{kg}], \dot{m}_{w f 2}$ - mass flow of water at inlet/outlet to/from feed water preheater $2[\mathrm{~kg} / \mathrm{s}], h_{w f 2^{\prime}}$ - specific enthalpy of water at inlet to feed water preheater $2[\mathrm{~kJ} / \mathrm{kg}], h_{w f 2}$ - specific enthalpy of water at outlet from feed water preheater 2 $[\mathrm{kJ} / \mathrm{kg}], h_{\text {waf } 2}$ - specific enthalpy of water after feed water preheater $2[\mathrm{~kJ} / \mathrm{kg}]$.

\section{Water pump before Feed water preheater_3}

$W_{p 3}=\dot{m}_{p f 2}\left[h_{w p f 3}-h_{w p f 2}\right]$

Mixer before Feed water preheater_3 energy balance.

$\dot{m}_{p f 2} h_{w f 3}+\dot{m}_{w f 2} h_{w a f 2}=\dot{m}_{w f 3} h_{w f 3}$

(26)Feed Water Preheater_3 energy balance:

$\dot{m}_{p f 3} h_{p f 3}+\dot{m}_{w f 3} h_{w f 3^{\prime}}=\dot{m}_{p f 3} h_{w f 4}+\dot{m}_{w f 3} h_{w a f 3}$

$\dot{m}_{p f 3}$ - mass flow of steam at inlet to feed water preheater $3[\mathrm{~kg} / \mathrm{s}], h_{p f 3}$ - specific enthalpy of steam at inlet to feed water preheater $3[\mathrm{~kJ} / \mathrm{kg}], \dot{m}_{w f 3}$ - mass flow of water at inlet/outlet to/from feed water preheater $3[\mathrm{~kg} / \mathrm{s}], h_{w f 3^{\prime}}$ - specific enthalpy of water at inlet to feed water preheater $3[\mathrm{~kJ} / \mathrm{kg}], h_{w f 4}$ - specific enthalpy of water at outlet from feed water preheater $3[\mathrm{~kJ} / \mathrm{kg}], h_{\text {waf } 3}$ - specific enthalpy of water after feed water preheater $3[\mathrm{~kJ} / \mathrm{kg}]$.

Water pump before Feed water preheater_4

$W_{p 4}=\dot{m}_{p f 4}\left[h_{w p f 5}-h_{w p f}\right]$

Mixer before Feed water preheater_4 energy balance.

$\dot{m}_{p f 3} h_{w f 5}+\dot{m}_{w f 3} h_{w a f 3}=\dot{m}_{w f 4} h_{w f 4}$

Feed Water Preheater_4 energy balance:

$\dot{m}_{p f 4} h_{p f 4}+\dot{m}_{w f 4} h_{w f 4^{\prime}}=\dot{m}_{p f 4} h_{w f 4}+\dot{m}_{w f 4} h_{w a f 4}$

$\dot{m}_{p f 4}$ - mass flow of steam at inlet to feed water preheater $4[\mathrm{~kg} / \mathrm{s}], h_{p f 4}$ - specific enthalpy of steam at inlet to feed water preheater $4[\mathrm{~kJ} / \mathrm{kg}], \dot{m}_{w f 4}$ - mass flow of water at inlet/outlet to/from feed water preheater $4[\mathrm{~kg} / \mathrm{s}], h_{w f 4^{\prime}}$ - specific enthalpy of water at inlet to feed water preheater $4[\mathrm{~kJ} / \mathrm{kg}], h_{w f} 4$ - specific enthalpy of water at outlet from feed water preheater $4[\mathrm{~kJ} / \mathrm{kg}], h_{\text {waf } 4}$ - specific enthalpy of water after feed water preheater $4[\mathrm{~kJ} / \mathrm{kg}]$.

\section{Water pump before deareator}

$W_{p 4}=\dot{m}_{p f 5}\left[h_{w p f 5}-h_{w p f 4}\right]$

Mixer before deareator energy balance.

$\dot{m}_{p f 4} h_{w f 5}+\dot{m}_{w f 4} h_{w a f 4}=\dot{m}_{w f 5} h_{w f 5}$

Deareator energy balance

$\dot{m}_{p d} h_{p d}+\dot{m}_{w f 5} h_{w f 5^{\prime}}+\dot{m}_{p p} h_{w p p}=\left[\dot{m}_{p p}+\dot{m}_{w f 5}+\dot{m}_{p d}\right] h_{w p d}$ 
$\dot{m}_{p d}$ - mass flow of steam at inlet to deareator $[\mathrm{kg} / \mathrm{s}], h_{p d}$ - specific enthalpy of steam at inlet to deareator $[\mathrm{kJ} / \mathrm{kg}], \dot{m}_{w f 5}$ - mass flow of water at inlet to deareator from mixer before deareator $[\mathrm{kg} / \mathrm{s}], h_{w f 5},-$ specific enthalpy of water at inlet to deareator from mixer before deareator $[\mathrm{kJ} / \mathrm{kg}], \dot{m}_{p p}$ - mass flow of water at inlet to deareator from control valve $[\mathrm{kg} / \mathrm{s}]$ $h_{w p p}$ - specific enthalpy of water at inlet from control valve $[\mathrm{kJ} / \mathrm{kg}], h_{w p d}$ - specific enthalpy of water deareator $[\mathrm{kJ} / \mathrm{kg}]$.

\section{Water pump after deareator}

$W_{p 5}=\left[\dot{m}_{p p}+\dot{m}_{w f 5}+\dot{m}_{p d}\right]\left[h_{w p d o}-h_{w p d}\right]$

$h_{\text {wpdo }}$ - specific enthalpy of water at the outlet from pump $[\mathrm{kJ} / \mathrm{kg}]$

\section{Total Efficiency}

$\eta_{T H 2}=\frac{P_{H P}+P_{M P}+P_{L P}-\sum W_{p i}+\eta H H V_{H 2}-Q u_{H 2}}{N_{R}}$

$P_{H P} / P_{M P} / P_{L P}$ High/Medium/Low-Pressure Steam Turbine Power [MW], $N_{R}$ - Reactor thermal power [MW], $Q_{u H 2}$ - Heat lost to heat up helium to hydrogen production [MW], $\eta$ - thermal efficiency of hydrogen production, $H H V$ - higher heating value $[\mathrm{kJ}] W_{p i}{ }^{-}$ work of water pump [MW]

\section{Model assumption}

High-temperature Nuclear Reactor coupled with heat recovery steam cycle is the main topic of present research for hydrogen and electricity production. Model assumptions for all simulation at this work and main thermodynamic parameters are described in Table 3.

Table 3. Model assumption and thermodynamic parameters.

\begin{tabular}{|c|c|c|}
\hline Main Parameter & Value & Unit \\
\hline High-Temperature Nuclear Reactor Power & 600 & $\mathrm{MW}_{\text {th }}$ \\
\hline Helium Pressure & 70 & $\mathrm{Bar}$ \\
\hline Helium Inlet Temperature & 235.9 & ${ }^{\circ} \mathrm{C}$ \\
\hline Helium Outlet Temperature & $800-940$ & ${ }^{\circ} \mathrm{C}$ \\
\hline HP/MP/LP Steam Turbine Isentropic Efficiency & 0,88 & - \\
\hline HP/MP/LP Steam Turbine Mechanical Efficiency & 0.998 & - \\
\hline Electrical Efficiency & 0,9856 & - \\
\hline MP Turbine first sink pressure & 29.4 & $\mathrm{Bar}$ \\
\hline MP Turbine second sink pressure & 10,5 & $\mathrm{Bar}$ \\
\hline MP Turbine third sink pressure & 4.8 & $\mathrm{Bar}$ \\
\hline LP Turbine first sink pressure & 1,92 & $\mathrm{Bar}$ \\
\hline LP Turbine second sink pressure & 0.24 & $\mathrm{Bar}$ \\
\hline LP Turbine third sink pressure & 0.08 & $\mathrm{Bar}$ \\
\hline Dearator inlet Pressure & 10.5 & $\mathrm{Bar}$ \\
\hline Condenser inlet Pressure & 0.08 & $\mathrm{Bar}$ \\
\hline Mass Flow for Water Cooling & 4707 & $\mathrm{~kg} / \mathrm{s}$ \\
\hline Helium Mas Flow Rate & 10 & $\mathrm{~kg} / \mathrm{s}$ \\
\hline Helium Inlet Pressure & 1 & $\mathrm{Bar}$ \\
\hline Helium Inlet Temperature & 20 & ${ }^{\circ} \mathrm{C}$ \\
\hline
\end{tabular}




\section{Results}

Results of the numerical simulations for HTGR system coupled with heat recovery steam cycle are presented in Figures 3-5. In the Fig.4(a) steam cycle in the h-s graph and in Fig. 3(b) superheated steam mass flow for different the reactor outlet temperature are presented.
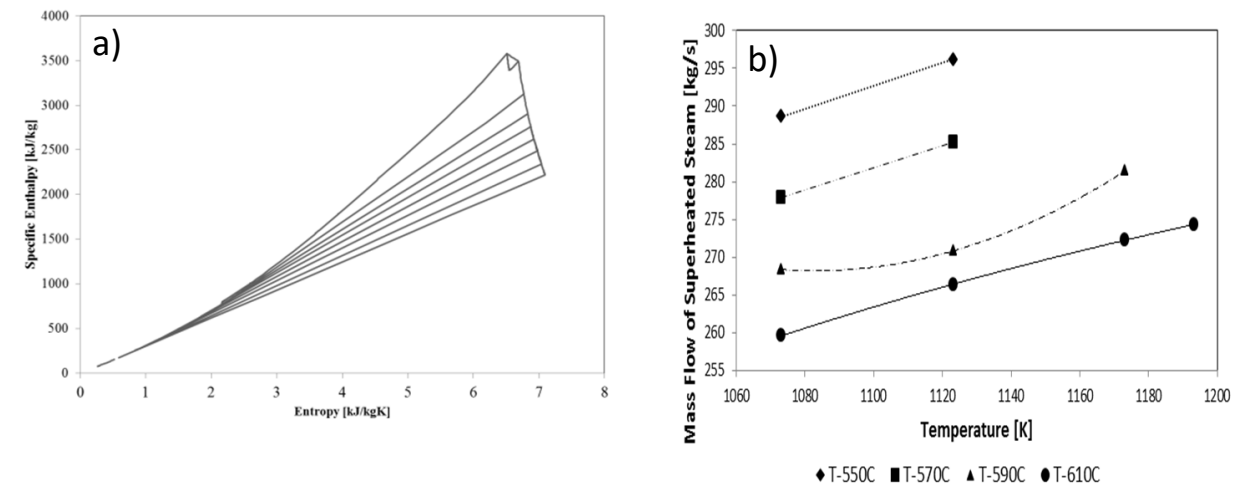

Fig. 3. h-s diagram (a) and superheated steam mass flow rate for different reactor outlet temperature (b).
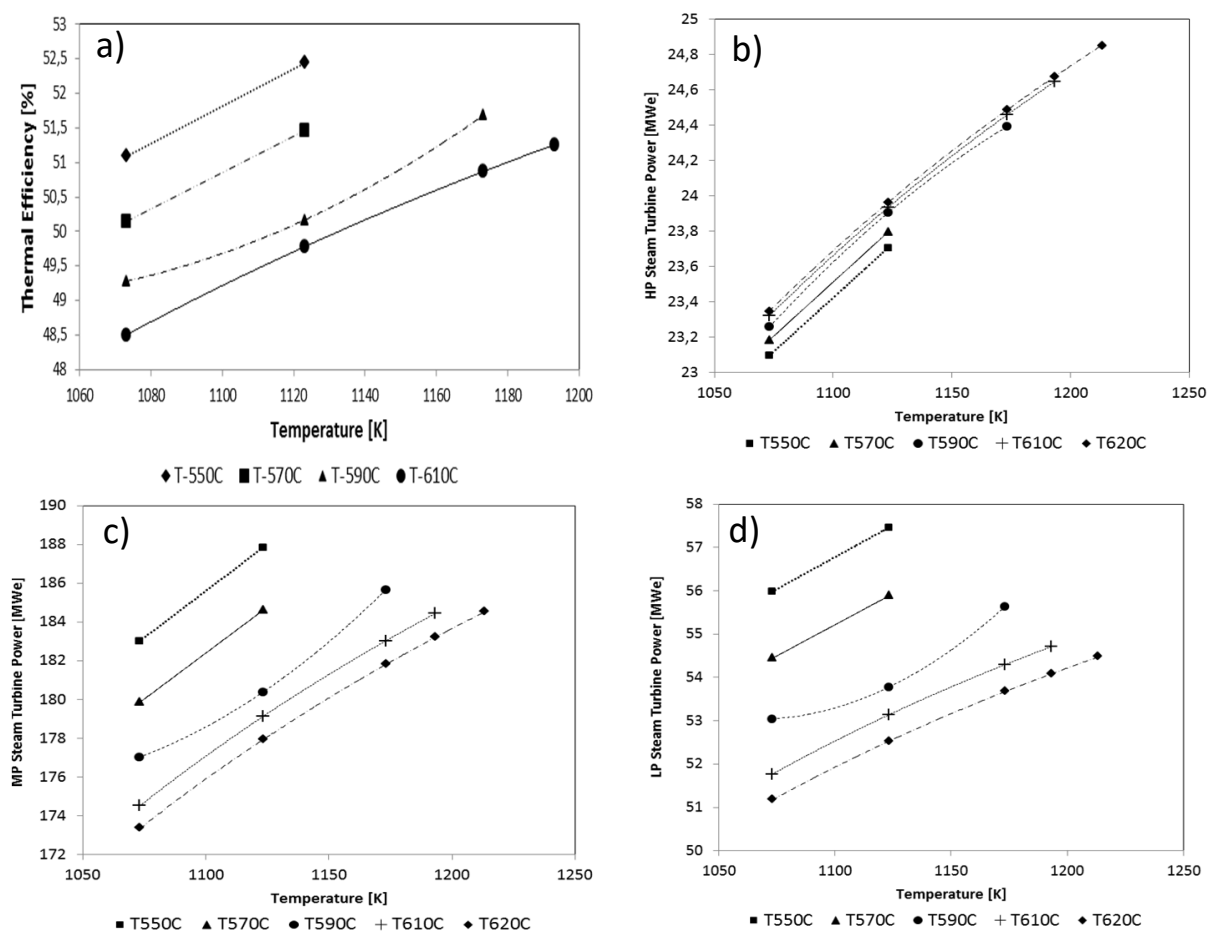

Fig.4. The thermal efficiency for heat recovery steam cycle without hydrogen production(a), HP-Steam turbine power at the various point of superheated steam (b), MP-Steam turbine power (c), LP-steam turbine power (d). 

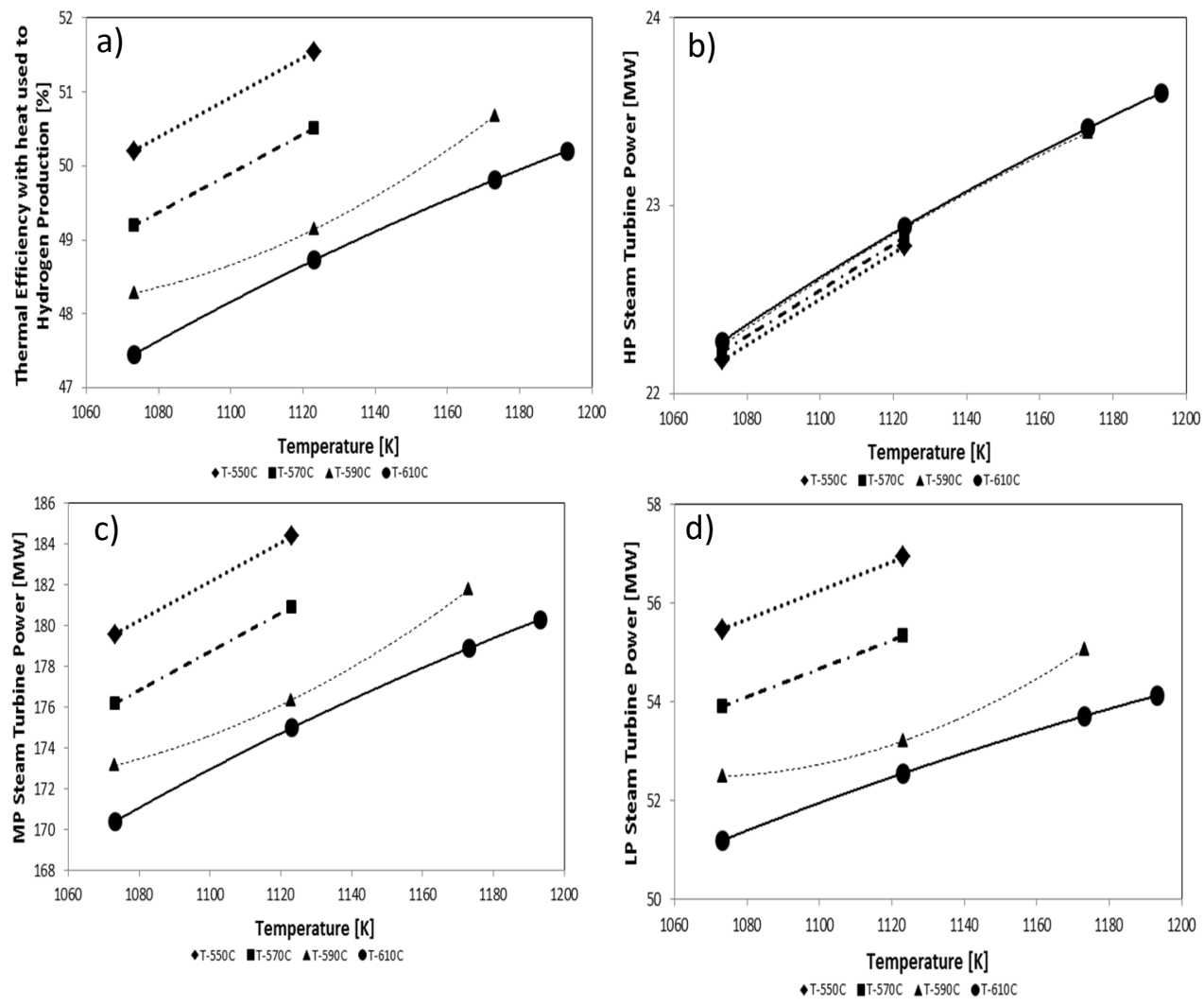

Fig.5. The thermal efficiency for heat recovery steam cycle with hydrogen production(a), HPSteam turbine power at the various point of superheated steam (b), MP-Steam turbine power (c), LP-steam turbine power (d).
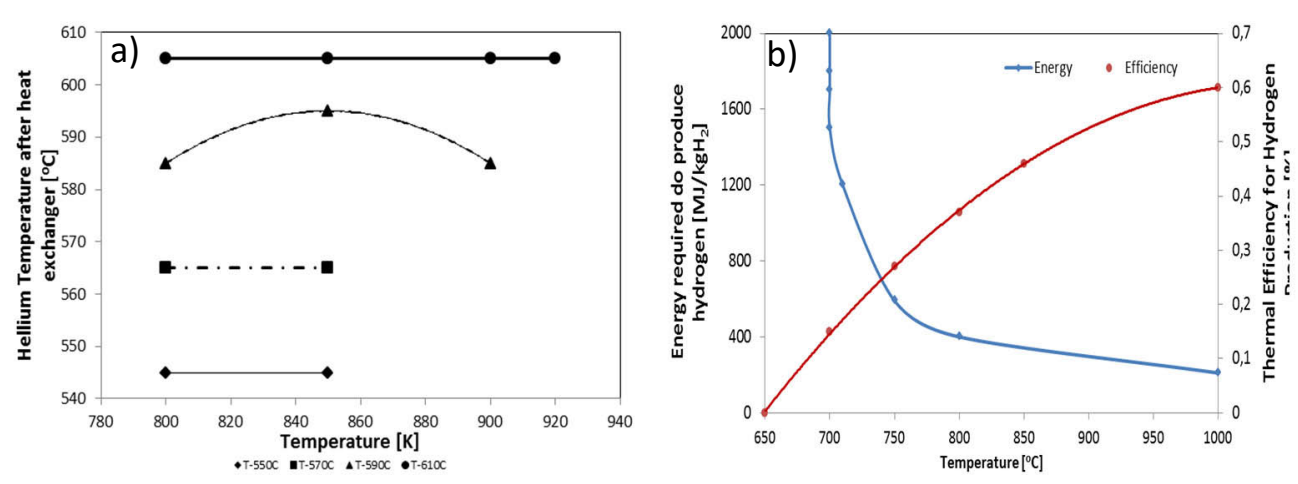

Fig.6. The graphs present (a) helium temperature to hydrogen process (b) thermal efficiency and thermal energy required to produce $1 \mathrm{~kg}$ of hydrogen using the MHR-SI [12]. 


\section{Conclusions}

High or very high-temperature nuclear reactors can be used to produce electricity with the high value of thermal efficiency. The power conversion system presented in Fig.1. and Fig. 2. has been analysed with and without hydrogen production in order to maximize the cycle efficiency. From a thermodynamic point of view, the most appropriate value of the thermal efficiency $52.5 \%$ is possible to achieve when cycle work in normal conditions for HighTemperature Nuclear Reactor and superheated steam should be superheated up to $530^{\circ} \mathrm{C}$ but when High-Temperature Nuclear Reactor coupled with heat recovery steam cycle works in supercritical conditions the value of thermal efficiency will be reduced by 2 to $3 \%$. The lowest value of thermal efficiency for cycle working in normal and supercritical conditions is respectively equal $51 \%$ and $48.5 \%$. When the outlet temperature from a nuclear reactor is increased up to $940^{\circ} \mathrm{C}$ it is possible to work in supercritical conditions what gives the opportunity to increase the value of the mass flow of superheated steam and increase a power from HP steam turbine, but the same time we can observe decrease the power of MP and LP steam turbine. The analysis shows that High-temperature Nuclear Reactor allows us effective realized much of industry process, give us the opportunity to produce hydrogen in a thermochemical cycle of coupled electricity generation and thermochemical hydrogen production process. What is more the analysis show that it is possible to achieve a high value of thermal efficiency with and without hydrogen production. The temperatures are not only one parameter to improve the thermal efficiency for High-Temperature. Another most important parameters are isentropic and mechanical efficiencies of the turbines and the compressor which has significant influence. High-temperature reactors used for electricity and hydrogen production have significant potential to improve efficiency by raising the reactor outlet temperature or steam temperature conditions.

The present work was supported by the Polish Ministry of Science (Grant AGH No.11.11.210.312).

\section{References}

1. S.J. Bae, J. Lee, Y. Ahn, J.I. Lee, Annals of Nuclear Energy 75 (2015)

2. U. Given, G. Velidi, Proceedings of International Congress on Advances in Nuclear Power Plants, ICAPP (2011)

3. B.J. Marsden, S.L. Fok, G. Hall, International Conference on Global Environment and Advanced Nuclear Power Plants (2003)

4. Generation IV Roadmap: R\&D Scope Report for Gas-Cooled Reactor Systems, Gen IV IF (2002)

5. Z. Zhang, Y. Dong, F. Li, Z. Zhang, H. Wang, X. Huang, Hong Li, B. Liu, X. Wu, H Wang, X. Diao, H. Zhang, J. Wang, 2 (2016)

6. Next Generation Nuclear Plant Research \& Development Program Plan, Idaho Nat Lab (2005)

7. International Prismatic Block HTGR Commercial Deployment Meeting Summary in Washington DC, March 8-10 (2016).

8. S.K. Mylavarapu, X. Sun, R. E. Glosup, R. N. Christensen, M.W. Patterson Applied Thermal Engineering 65 (2014)

9. Y. Shin, T.Lee, K. Lee, M. Kim Applied Energy 173 (2016)

10. G. Jezierski, Conventional and Nuclear Power [in Polish], Energetyka Cieplna i Zawodowa 10 (2009)

11. C.F. McDonald, Appl Thermal Eng 73 (2014)

12. B.Yildiz, M. S. Kazimi, International Journal of Hydrogen Energy 31 (2006) 